\section{Somatic reversion events point towards RPL4 as a novel disease gene in a condition resembling Diamond-Blackfan anemia}

Somatic revertant mosaicism is a rare phenomenon caused by spontaneous correction of a pathogenic allele and subsequent clonal expansion of the revertant cell. It explains patches of healthy skin in conditions like epidermolysis bullosa and improvement or absence of haematological conditions such as bone marrow failure in Fanconi anemia, dyskeratosis congenita and the recently identified syndromes caused by mutations in $S A M D 9$ and $S A M D 9$. $^{1-5}$ Revertant mosaicism is restricted to conditions that fulfil two prerequisites: the diseased cells have the ability to replicate, and loss of the mutation gives these cells a selection advantage compared to surrounding mutated cells. Awareness of revertant mosaicism is relevant to understanding variable expression of syndromes and to understanding the absence of an expected mutation in DNA diagnostics.

A common cause of revertant mosaicism in dominant disorders is mitotic recombination, resulting in a daughter cell with two paternal copies of a chromosome segment and one with two maternal copies. Clonal expansion of the cells lacking the mutation relative to the mutated cells results in mosaic segmental uniparental disomy (UPD), which can be easily visualized in the B-allele frequency plot of SNP arrays, even when present in a subset of the cells. ${ }^{1}$ Acquired UPD observations can assist in disease gene identification, as was illustrated for the condition Ichthyosis with confetti. ${ }^{6}$

Herein, we report about a girl who was born small for gestational age (2440 gr. at term; <-2,5SD), after an uneventful pregnancy. She had a unilateral partial cleft lip, low set ears and toe anomalies consisting of bilateral shortening of the middle phalanges of the $2^{\text {nd }}-4^{\text {th }}$ toe and duplication of the distal phalanges of these toes (Online
A

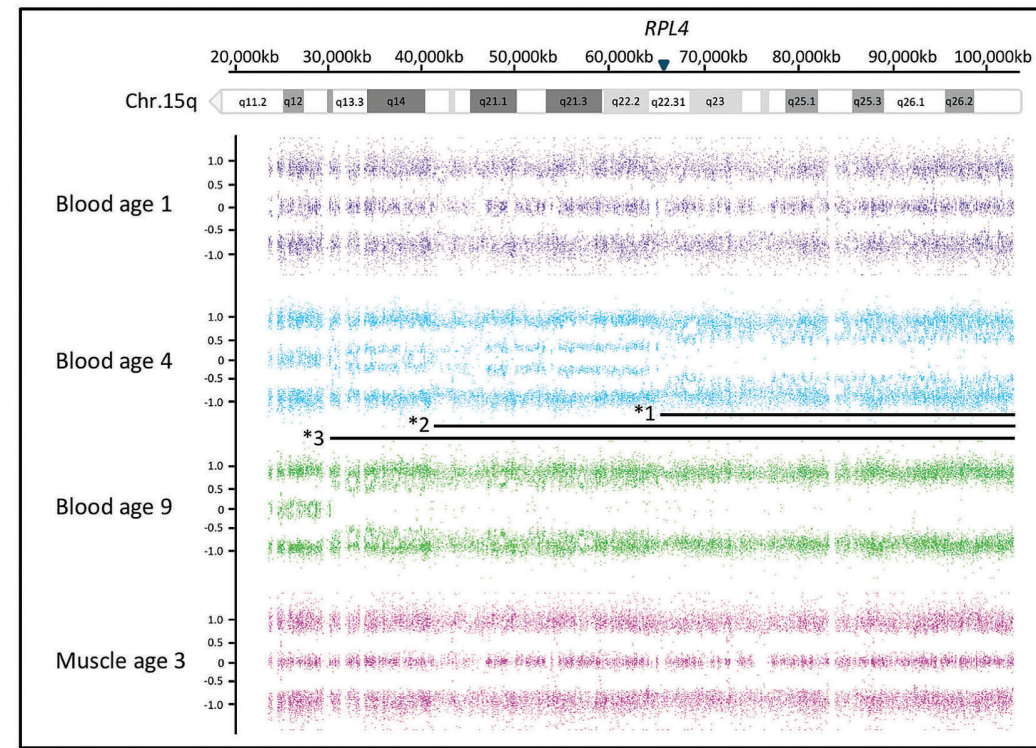

B

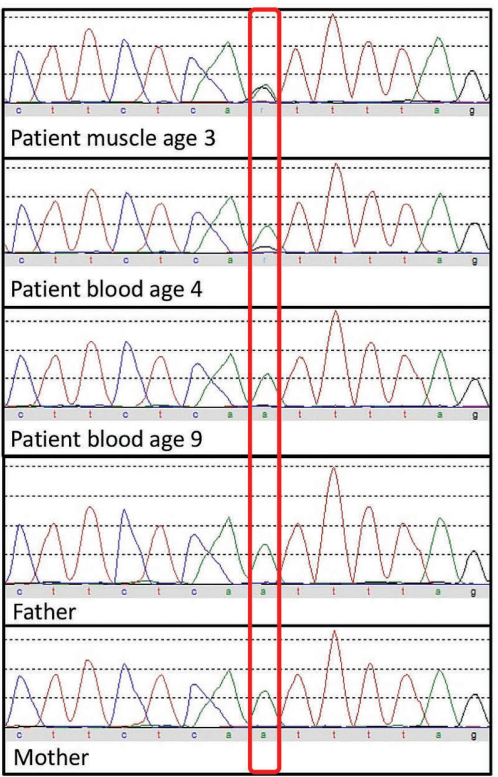

C Exon 2

gaAuUagcagGucaucagacuagugcu

Exon 3

Exon 2

gaAuUagcag JuUuagutaucagacu

Exon 3
Figure 1. Acquired 15qUPD assists in the identification of an RPL4 mutation. (A) CytoscanHD B-allele frequency plots of chr. 15 of DNA isolated from blood samples drawn at increasing ages and from a muscle biopsy. A blood sample drawn at age 4 shows three stretches of acquired mosaic UPD, with breakpoints at $\sim 64.5 \mathrm{Mb}$ in $15 \mathrm{q} 22.31, \sim 40 \mathrm{Mb}$ in $15 q 14$, and $\sim 29.5 \mathrm{Mb}$ in $15 \mathrm{q} 13.1$, respectively $(* 1, * 2$ and $* 3)$. The samples taken at an earlier (age 1) and later (age 9) time point show a normal B-allele frequency pattern on $15 q$ in the first year of life, and complete $15 q$ homozygosity at age 9 . In muscle-derived DNA, a normal B-allele frequency pattern is observed. (B) Sanger sequencing analysis of the identified mutation in RPL4 (indicated in the red box) in the patient and the parents (FW sequences). In the muscle biopsy, the mutation is present in a heterozygous state. In the blood sample drawn at age 4, the mutation is present in a mosaic state ( $20 \%)$. In the blood sample drawn at age 9 , the mutation can no longer be identified. The sequencing analysis showed that the mutation is absent in the father and the mother. (C) Schematic mRNA sequence of the exon 2-3 boundary of wild type (WT) and mutant (MUT) RPL4, with the inserted nucleotides in pink. 
Supplementary Figure S1). In her first year of life, hypotonia and developmental delay were observed. At 11 months of age, she was admitted to the hospital due to failure to thrive. Hepatosplenomegaly was noted and a routine full blood count showed mild anemia (hemoglobin $5.7 \mathrm{mmol} / \mathrm{l}$ (normal 7.5-10.0 mmol/L), mean corpuscular volume 79 fL). A metabolic disease was suspected, for which extensive metabolic testing was performed and revealed no abnormalities. Since she showed no clinical signs of severe anemia, hemoglobin count was never followed up and the hepatosplenomegaly improved over time. Later in childhood, clinical signs of anemia were not observed, and complete blood counts and $\mathrm{Hb}$ testing were not performed anymore. The girl had a borderline intellectual disability with attention deficit disorder and attends a special school. At ten years of age, she had short stature $(123 \mathrm{~cm},<-$ $2,5 \mathrm{SD})$ and unilateral hearing loss due to frequent otitis. Informed consent for publication of clinical information and photographs was obtained from parents.

In search of a genomic abnormality underlying this phenotype, a routine diagnostic genome-wide SNP array analysis was performed on blood and muscle derived DNA (CytoscanHD; Life Technologies, Carlsbad, CA 92008 USA). With a genome-wide SNP array on blood sampled at age 4, no copy number variations explaining the phenotype of the girl were identified, but along chromosome $15 \mathrm{q}$ towards the telomer, at least three flanking regions with increasing imbalances in B-allele frequency were encountered, suggestive of multiple subclonal mitotic reversion events resulting in acquired mosaic UPD15qter (Figure 1A).

We hypothesized that these clonal UPD events resulted from a selective advantage of cells in which an as of yet unidentified heterozygous mutation, potentially explaining the symptoms in our patient, had reverted to wild type. Indeed, SNP array analysis on blood DNA samples taken at an earlier (age 1) and later (age 9) time point revealed progression from a normal B-allele frequency pattern on $15 q$ in the first year of life towards complete $15 q$ homozygosity at nine years of age (Figure 1A). SNP array analysis on DNA derived from a muscle biopsy taken as a diagnostic procedure when the patient was three years old did not show UPD15qter (Figure 1A), illustrating the tissue-specificity of this phenomenon.

The emerging $15 q$ homozygosity in blood cells overlapped with the disappearing features of anemia, suggesting that the region distal from the most telomeric recombination breakpoint at $15 \mathrm{q} 22.31$ carries a genetic mutation
A

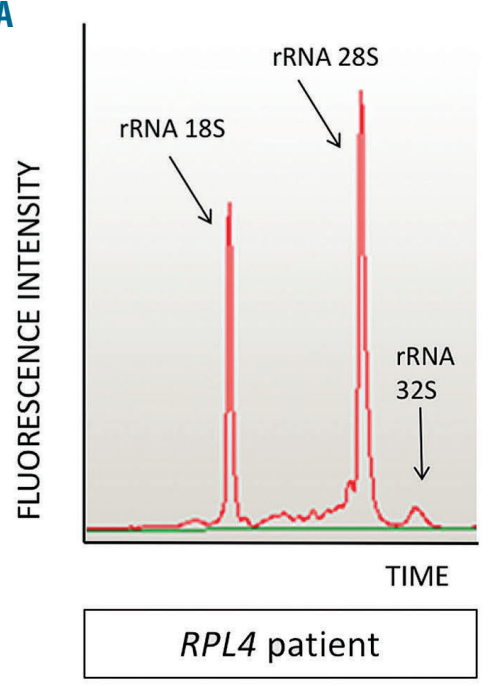

C

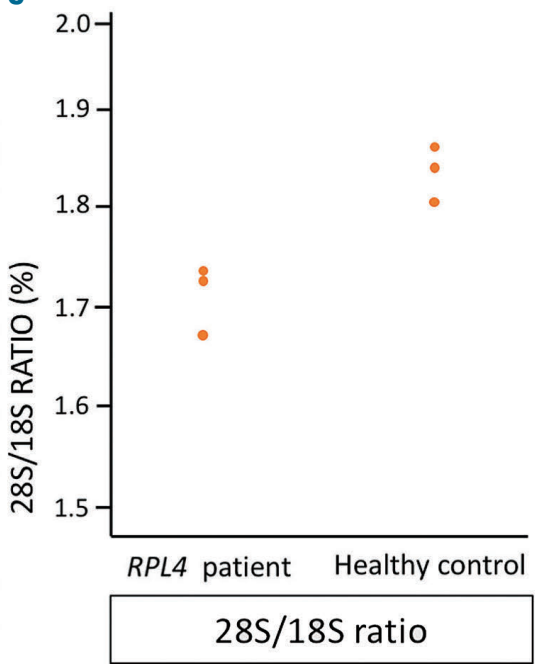

B

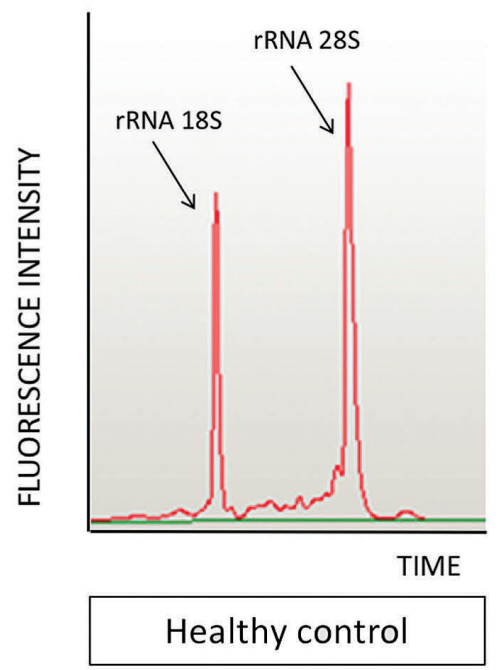

D

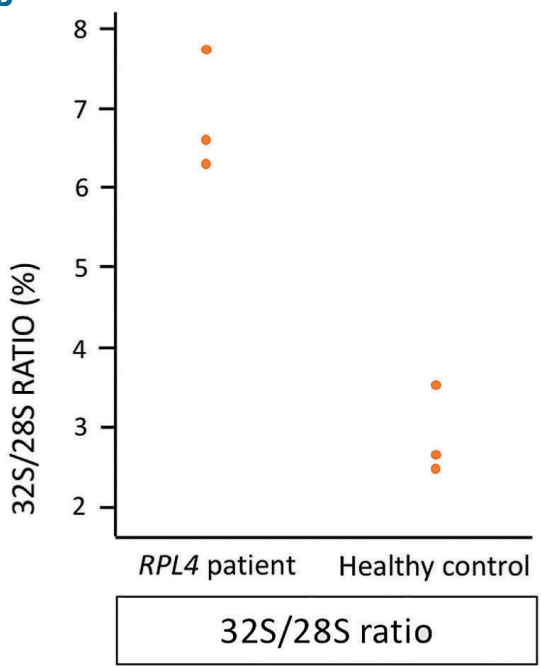

Figure 2. Impaired rRNA processing in the RPL4-mutated patient. Agilent Bioanalyser 2100 electropherograms obtained from total RNA of fibroblasts taken at age 3 of the patient carrying a RPL4 mutation $(A)$ and from a healthy control (B). The electropherogram data demonstrated two prominent peaks corresponding to $18 \mathrm{~S}$ and $28 \mathrm{~S}$ rRNA. Based on the quantified peak surfaces, we detected a reduced $28 \mathrm{~S} / 18 \mathrm{~S}$ ratio (C) and increased $32 S / 28 S$ ratio (D) of the patient compared to the healthy control. Depicted are the values determined in three independent measurements. 
with growth disadvantage in the hematopoietic system. Whole exome sequencing was performed on muscle derived DNA (WES; SureSelect All Exon DNA enrichment; Solid 5500 XL sequencing). The region distal of 15q22.31 contains five OMIM genes that could possibly explain (part of) the phenotype of the patient. However, none of these genes carried mutations (Online Supplementary Table S1). A search for rare coding variants with allele frequencies in $\mathrm{dbSNP}$ and gnomAD $<1 \%$ revealed four potential candidate genes (Online Supplementary Table S2). One of these variants was a de novo mutation (c.176-7A>G) in RPL4 (MIM: 180479; NM_000968.3; Figure 1B), a ribosomal protein gene highly intolerant for mutations (ExAC pLI score $=1.00)$. Sanger sequencing confirmed that this mutation was present in a full heterozygous state in muscle derived DNA, subclonal ( 20\%) in the blood sample at age 4, and completely absent in blood at age 9 (Figure 1B). The variant identified had not been reported before in our in-house database, nor in the databases ExAC, gnom $A D$, GoNL or the Exome Variant Server (ESP).

Sequencing of cDNA from fibroblast-derived RPL4 RNA of the patient showed that the c.176-7A $>$ G variant affects splicing, creating a six-nucleotide insertion (r.175_176insUUUUAG; p.(Ala58_Gly59insValLeu); Figure 1C) in the transcript, which was not affected by nonsense-mediated decay (NMD; Online Supplementary Figure S2).

The RPL4 gene encodes ribosomal protein L4. So far, mutations in RPL4 have not been linked to human diseases, but mutations in other ribosomal protein (RP) genes cause Diamond-Blackfan anemia (DBA). ${ }^{7}$ A substantial portion of patients with a clinical diagnosis of DBA remain genetically unexplained. ${ }^{8} \mathrm{DBA}$ is mainly characterized by chronic hyporegenerative anemia, and approximately $50 \%$ of patients with DBA have additional anomalies, including developmental delay, short stature, cleft lip and low set ears. These features overlap with the symptoms described in our patient. The most frequent skeletal anomaly in DBA are thumb defects, but anomalies of the toes as identified in our patient have also been reported.

Next, we studied whether this splice site mutation disrupts ribosome biogenesis similar to other DBA causing RP gene mutations. Ribosome biogenesis involves the assembly of RPs and ribosomal RNAs (rRNAs), which together comprise the structural components of the $40 \mathrm{~S}$ and $60 \mathrm{~S}$ ribosomal subunits. In patients with mutated RPs of the large ribosomal subunit (RPLs), characteristic pre-rRNA processing signatures are found, characterized by lower $28 \mathrm{~S} / 18 \mathrm{~S}$ ratios and higher residual levels of the $32 \mathrm{~S}$ precursor. ${ }^{10}$

We analysed rRNA processing using capillary electrophoresis analysis of rRNA maturation of the 40S and $60 S$ ribosomal subunits as described before. ${ }^{10}$ Ribosomal RNA processing analysis on our patient's fibroblasts in which the mutant allele is expressed, showed a reduced $28 \mathrm{~S} / 18 \mathrm{~S}$ ratio and an increased $32 \mathrm{~S}$ precursor peak compared to a healthy control (Figure 2). These results suggest an impairment of rRNA processing resulting from the RPL4 splice site mutation in fibroblasts of our patient, similar to what is observed for other DBA-causing RP genes.

Recently, another example of revertant mosaicism in a patient with DBA, based on a deletion encompassing $R P S 26$, was reported. ${ }^{11}$ In the patient described here, we observed three separate events of mitotic recombination resulting in acquired mosaic UPD15q-tel. This indicates that these events provoke a selection advantage resulting in clonal outgrowth and replacement of RPL4 mutated cells. Somatic reversion events in blood may occur more frequently in DBA as, in $20 \%$ of these patients, the anemia goes in remission. ${ }^{12,13}$ Such events hamper the identification of mutations in blood, which may explain why mutations in RPL4 have not previously been reported. ${ }^{8,14}$

In conclusion, we here report the occurrence of mitotic recombination-based somatic reversion in a girl with a Diamond-Blackfan anemia-like phenotype and show how this genetic event directed us to identify RPL 4 as a novel disease-causing gene.

Marjolijn C. J. Jongmans, ${ }^{1,2,3}$ Illja J. Diets, ${ }^{1}$ Paola Quarello, ${ }^{4}$ Emanuela Garelli, Roland P. Kuiper ${ }^{3}$ and Rolph Pfundt

${ }^{1}$ Department of Human Genetics, Radboud university medical center and Radboud Institute for Molecular Life Sciences, Nijmegen, the Netherlands; 'Department of Medical Genetics, University Medical Center Utrecht, the Netherlands; ${ }^{3}$ Princess Máxima Center for Pediatric Oncology, Utrecht, the Netherlands; ${ }^{4}$ Paediatric Onco-Haematology, Stem Cell Transplantation and Cellular Therapy Division, Regina Margherita Children's Hospital, Torino, Italy and 'Department of Public Health and Paediatric Sciences, University of Torino, Italy

Funding: Illja J. Diets is funded by the KiKa Foundation (project 127).

Correspondence:M.C.J.Jongmans-3@umcutrecht.nl doi:10.3324/haematol.2018.200683

Information on authorship, contributions, and financial \& other disclosures was provided by the authors and is available with the online version of this article at www. haematologica.org.

\section{References}

1. Jongmans MC, Verwiel ET, Heijdra Y, et al. Revertant somatic mosaicism by mitotic recombination in dyskeratosis congenita. Am J Hum Genet. 2012;90(3):426-433.

2. Jonkman MF, Scheffer H, Stulp R, et al. Revertant mosaicism in epidermolysis bullosa caused by mitotic gene conversion. Cell. 1997;88(4):543-551

3. Waisfisz Q, Morgan NV, Savino M, et al. Spontaneous functional correction of homozygous fanconi anaemia alleles reveals novel mechanistic basis for reverse mosaicism. Nat Genet. 1999;22(4):379383.

4. Bluteau $\mathrm{O}$, Sebert $\mathrm{M}$, Leblanc $\mathrm{T}$, et al. A landscape of germ line mutations in a cohort of inherited bone marrow failure patients. Blood. 2018;131(7):717-732.

5. Tesi B, Davidsson J, Voss M, et al. Gain-of-function SAMD9L mutations cause a syndrome of cytopenia, immunodeficiency, MDS, and neurological symptoms. Blood. 2017;129(16):2266-2279.

6. Choate KA, Lu Y, Zhou J, et al. Mitotic recombination in patients with ichthyosis causes reversion of dominant mutations in KRT10. Science. 2010;330(6000):94-97

7. Clinton C, Gazda HT. Diamond-Blackfan Anemia. In: Pagon RA, Adam MP, Ardinger $\mathrm{HH}$, et al, eds. GeneReviews(R). Seattle (WA), 1993.

8. Mirabello L, Khincha PP, Ellis SR, et al. Novel and known ribosomal causes of Diamond-Blackfan anaemia identified through comprehensive genomic characterisation. J Med Genet. 2017 Jun;54(6):41725.

9. Errichiello E, Vetro A, Mina T, et al. Whole exome sequencing in the differential diagnosis of Diamond-Blackfan anemia: clinical and molecular study of three patients with novel RPL5 and mosaic RPS19 mutations. Blood Cells Mol Dis. 2017;64:38-44.

10. Quarello P, Garelli E, Carando A, et al. Ribosomal RNA analysis in the diagnosis of Diamond- Blackfan Anaemia. Br J Haematol. 2016;172(5):782-785.

11. Venugopal P, Moore S, Lawrence DM, et al. Self-reverting mutations partially correct the blood phenotype in a Diamond Blackfan anemia patient. Haematologica. 2017 ;102(12):e506-e509.

12. Chen S, Warszawski J, Bader-Meunier B, et al. Diamond-blackfan anemia and growth status: the French registry. J Pediatr. 2005; 147(5):669-673.

13. Vlachos A, Ball S, Dahl N, et al. Diagnosing and treating Diamond Blackfan anaemia: results of an international clinical consensus conference. Br J Haematol. 2008;142(6):859-876.

14. Doherty L, Sheen MR, Vlachos A, et al. Ribosomal protein genes RPS10 and RPS26 are commonly mutated in Diamond-Blackfan anemia. Am J Hum Genet. 2010;86(2):222-228. 